\title{
VALIDATION OF THE POLARIS CANDU EXTENSION FOR LATTICE PHYSICS
}

\author{
Simon Younan ${ }^{1}$, Dr. David Novog ${ }^{1}$ \\ ${ }^{1}$ McMaster University \\ 1280 Main Street West \\ Hamilton, Ontario, Canada, L8S 4L7 \\ younans@mcmaster.ca,novog@mcmaster.ca
}

\begin{abstract}
Polaris is a new lattice physics package, introduced in version 6.2 of the SCALE package. It uses a method of characteristics transport solver and the embedded self-shielding method. It is able to model light water reactor systems with a minimal amount of input. The goal of this project is to include support for CANDU models in Polaris for the next version of SCALE. So far, the model has been implemented and shown to give results with reasonable agreement to other SCALE sequences. This study extends the model to a reflector model, and shows that most quantities agree well with other codes. Some quantities, such as $\mathrm{k}_{\mathrm{eff}}$ and assembly discontinuity factors, are sensitive to meshing. This study also performs a correlation between the TRITON and Polaris sequences using Sampler to perturb the nuclear data. Overall, there is good agreement between the two codes, though coolant void reactivity is only moderately correlated, likely due to the differences in resonance self-shielding methods. Additionally, this work shows that a coarser mesh can be used to speed up uncertainty calculations compared to the mesh used for a best estimate. Finally, this work shows that the mass lumping feature in CENTRM significantly affects heavy water moderated calculations, whether using TRITON or calculating self-shielding factors, and thus should be disabled for heavy water calculations.
\end{abstract}

KEYWORDS: lattice physics, CANDU, method of characteristics, embedded self-shielding method, uncertainty analysis

\section{INTRODUCTION}

Polaris ${ }^{1}$ is a recently-developed lattice physics code introduced to the SCALE code system in version 6.2 [1] for light water reactor lattice physics analysis. It uses the method of characteristics to solve the transport equation. Its unique feature is the implementation of the embedded self-shielding method (ESSM). This method is derived from the Bondarenko method through a clever application of equivalence theory [2]. The requirement to calculate an equivalent lattice cell or Dancoff factor is eliminated, in contrast to the TRITON sequence. Additionally, the need for a time-consuming pointwise transport solution, as is done by CENTRM for resonance self-shielding, is eliminated, instead only requiring independent one-group solutions for each energy group in the multi-group library. Resonance self-shielding is then performed by interpolating a table of self-shielding factors.

\footnotetext{
${ }^{1}$ Not to be confused with the Japanese core management code POLESTAR, which bears no relation to Polaris.
} 
Most reactor physics codes permit a generalized specification of the geometry, where the user defines individual surfaces in the geometry, usually with some optimizations such as being able to specify arrays and nested geometries. Polaris takes a more constrained approach, where it assumes that the user wants to model a particular well-known system and can specify parameters for that system. Currently, Polaris only supports PWR and BWR geometries [2]. The motivation for this project is to extend the code to allow its use for other reactor systems, with this project focusing on the CANDU reactor, a Canadiandesigned pressure tube heavy water reactor. In addition to extending the capabilities of the code, the selfshielding and transport calculations shall be validated to show that the code gives results which are comparable to existing codes and experimental results.

\section{BACKGROUND AND PRIOR WORK}

\subsection{Polaris ESSM Theory}

The most unique aspect of Polaris is its implementation of the embedded self-shielding method (ESSM) as an alternative to the standard Bondarenko method (through BONAMI) and the Continuous-Energy Transport Module (CENTRM) available to other SCALE modules. The Bondarenko method uses the intermediate-resonance (IR) approximation to compute self-shielded cross-sections as a function of the non-resonant (background) cross-section [1]. This relationship is tabulated during library generation, with the SCALE distribution using light water reference models computed by CENTRM. For a heterogeneous model, equivalence theory is applied to calculate an "escape cross-section" contribution, based on the Dancoff factor for the lattice geometry [1].

In Polaris, ESSM uses the Bondarenko method, but applies the equivalence theory mathematically to the IR-approximated transport equation, i.e. Equation (1) [2].

$$
\left(\widehat{\Omega} \cdot \nabla+\Sigma_{t, g}(\bar{r})\right) \psi_{g}(\bar{r}, \widehat{\Omega})=\frac{1}{4 \pi} \sum_{i} \sigma_{p, i} N_{i}(\bar{r})\left(\left(1-\lambda_{g, i}\right) \phi_{g}(\bar{r})+\lambda_{g, i} \Delta u_{g}\right)
$$

This equation may be solved by any transport solver - Polaris uses the same transport solver as for the main transport calculation. This solution is very quick to compute, as Equation (1) is a one-group equation. Equivalence theory replaces the transport term with the escape cross-section, which acts as an additional scattering material. This produces an algebraic equation which can be simplified to isolate the escape cross-section [2]:

$$
\Sigma_{e s c, g}(\bar{r})=\frac{\Sigma_{a, g}(\bar{r}) \phi_{g}(\bar{r})}{\Delta u_{g}-\phi_{g}(\bar{r})}-\sum_{i} \lambda_{g, i} \sigma_{p, i} N_{i}(\bar{r})
$$

The flux from solving Equation (1) is used to calculate the escape cross-section in Equation (2) for each mesh cell. These are used in the Bondarenko method to calculate background cross-sections for selfshielding, directly using the problem geometry and avoiding the need to approximate it with a Dancoff factor. However, the above equations should be solved using self-shielded cross-sections, thus an iterative method is used, where these steps are repeated until the solution converges [2].

The validity of applying the self-shielding factors from a light water reference to non-light-water systems is a topic up for investigation by this project. 


\subsection{Prior Work on this Project}

The implementation of a CANDU geometry model in Polaris was straightforward. The bulk of the project involved a code-to-code comparison between Polaris and other SCALE sequences, along with an investigation of generating alternative self-shielding factors, particularly using heavy water. The Monte Carlo code KENO, in continuous-energy mode, was used as the reference calculation for most cases.

This project has looked at $\mathrm{k}_{\infty}$, depletion, reactivity coefficient, and group constant comparisons, along with a sensitivity study to investigate the effect of self-shielded cross-section differences, and uncertainty analyses using Sampler, all for a CANDU model. A benchmark comparison with the ZED-2 experiment [3] had also been performed. Overall, the results are promising, and the work has been published as an article in Nuclear Engineering \& Design [4]. The work described in this paper is a continuation of that work, following up on issues discovered during the analysis, along with performing additional comparisons, which are described in Section 3.

\section{METHODOLOGY}

\subsection{Reflector model comparison}

Support for a reflector model for the CANDU cluster builder was added to Polaris, and a model created in both Polaris and NEWT, as well as in DRAGON5 [5] as an additional reference. Comparison of the reflector group constants and assembly discontinuity factors was performed. The calculation was performed using mid-burnup fuel compositions. The model, for Polaris, is shown in Fig. 1. Sampler was executed on the Polaris model to determine the uncertainty due to nuclear data.

The DRAGON model uses the subgroup method for self-shielding and a 361-group energy structure [6].

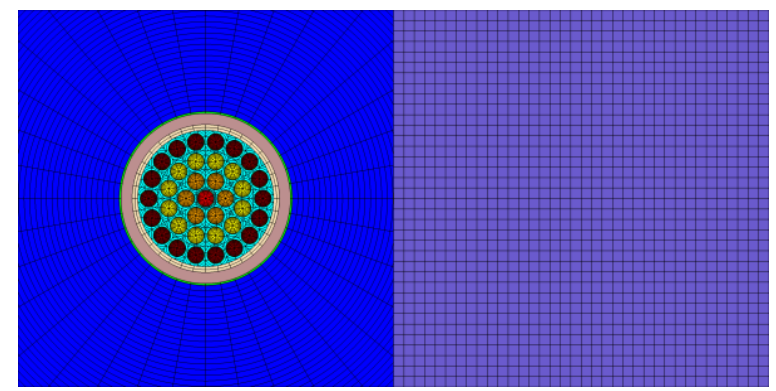

Figure 1. CANDU Lattice Model with Reflector

In addition to the code comparison, a mesh sensitivity analysis was performed on the reflector region to determine the effect of the reflector meshing on the results, along with whether the reflector mesh density in Fig. 1 is adequate. The meshing in the fuel channel region is left unchanged.

\subsection{Correlation study using Polaris and TRITON-NEWT with Sampler}

To study the similarity of the results between the two codes, a correlation study was performed using Sampler. When using Sampler, cases with the same sample number have the same perturbation, thus there should be a correlation between the two cases based upon the similarity of the models. If the two models are identical, the results for a particular quantity shall be identical, and thus both perfectly correlated with a slope equal to unity. When the same system is modelled in both cases, any differences 
are attributed to the precision of the model (e.g. mesh density) and differences in the approximations present in the calculation methods.

Two sets of Sampler calculations were compared. The first compares Polaris and TRITON-NEWT. The second looks at two Polaris models that differ only in mesh density, with one model having a significantly coarser mesh.

\subsection{Investigation of CENTRM mass lumping for self-shielding factors}

For this procedure, the mass lumping optimization (alump) in CENTRM was toggled between the default setting (alump=0.2) and being disabled (alump=0). This was done for the $\mathrm{D}_{2} \mathrm{O}$ pin cell irffactor calculation, an $\mathrm{H}_{2} \mathrm{O}$ pin cell irffactor calculation, as well as the TRITON calculations using CENTRM. The transport and depletion calculations were performed on these models and the results compared to those from the originally distributed self-shielding factors, as well as those from the homogeneous models. The sensitivity of $\mathrm{k}_{\infty}$ and reaction rates to the self-shielded cross-sections was computed using TSUNAMI, in order to compute biases due to the self-shielded cross-sections themselves.

\section{RESULTS AND DISCUSSION}

\subsection{Reflector model comparison}

Table 1 shows that, except for assembly discontinuity factors, the difference between the two codes for a reflector model is smaller than the nuclear data uncertainty. However, both $k_{\text {eff }}$ and the assembly discontinuity factors are particularly sensitive to the mesh density, especially in NEWT. NEWT is much more sensitive to meshing variations in both dimensions, as shown by Fig. 2. Both codes are sensitive to horizontal meshing, while NEWT is also sensitive to vertical meshing.

When the results from the finest meshes shown in Fig. 2 are used, the biases in the ADFs shown in Table 1 decrease to nearly zero, and the bias in $k_{\text {eff }}$ is reduced substantially, from nearly $770 \mathrm{pcm}$ down to $150 \mathrm{pcm}$. Too fine of a mesh, however, is costly in CPU and memory requirements, especially for NEWT. In this particular case, Polaris can produce accurate results with a coarser mesh than NEWT.

The $\mathrm{k}_{\text {eff }}$ bias of Polaris, compared to DRAGON, is $150.0 \mathrm{pcm}$, putting the Polaris $\mathrm{k}_{\text {eff }}$ closer to DRAGON than the NEWT $k_{\text {eff. }}$ Total and absorption cross-sections are in good agreement, while the downscattering cross-sections is about 7\% greater for DRAGON, possibly due to the differences in the energy group structure.

Table 1. Comparison of reflector group constant biases

\begin{tabular}{|c|c|c|c|c|c|c|}
\hline Quantity & \multicolumn{2}{|c|}{$\begin{array}{c}\text { Polaris minus NEWT } \\
\text { (abs/rel) }\end{array}$} & \multicolumn{2}{|c|}{$\begin{array}{l}\text { Polaris minus DRAGON } \\
(\mathrm{abs} / \mathrm{rel})\end{array}$} & \multicolumn{2}{|c|}{$\begin{array}{c}\text { Sampler Standard Deviation } \\
\text { (abs/rel) }\end{array}$} \\
\hline $\mathrm{k}_{\text {eff }}$ & \multicolumn{2}{|c|}{$+768.6 \mathrm{pcm}$} & \multicolumn{2}{|c|}{$-150.0 \mathrm{pcm}$} & \multicolumn{2}{|c|}{$1150 \mathrm{pcm}$} \\
\hline $\mathrm{k}_{\mathrm{eff}}$ (finest mesh) & \multicolumn{2}{|c|}{$+149.2 \mathrm{pcm}$} & & & & \\
\hline $\mathrm{ADF}_{1}$ & +0.012 & $+1.22 \%$ & & & 0.0019 & $0.19 \%$ \\
\hline $\mathrm{ADF}_{2}$ & +0.0062 & $+2.25 \%$ & & & 0.0029 & $1.04 \%$ \\
\hline$\Sigma_{\mathrm{a} 2}\left(\times 10^{-6} \mathrm{~cm}^{-1}\right)$ & -0.17 & $-0.21 \%$ & -0.54 & $-0.66 \%$ & 1.02 & $1.24 \%$ \\
\hline$\sum_{\mathrm{a} 1}\left(\times 10^{-6} \mathrm{~cm}^{-1}\right)$ & -0.4447 & $+25.03 \%$ & -1.84 & $* * * * * *$ & 12.75 & $* * * * * *$ \\
\hline$\sum_{\mathrm{tr} 2}\left(\times 10^{-3} \mathrm{~cm}^{-1}\right)$ & -0.0 & $-0.01 \%$ & & & 6.2 & $1.52 \%$ \\
\hline$\sum_{\mathrm{tr} 1}\left(\times 10^{-3} \mathrm{~cm}^{-1}\right)$ & -0.8 & $-0.31 \%$ & & & 3.2 & $1.23 \%$ \\
\hline$\sum_{1 \rightarrow 2}\left(\times 10^{-3} \mathrm{~cm}^{-1}\right)$ & -0.08 & $-0.47 \%$ & -1.29 & $-6.77 \%$ & 0.37 & $2.11 \%$ \\
\hline$\Sigma_{2 \rightarrow 1}\left(\times 10^{-6} \mathrm{~cm}^{-1}\right)$ & -0.10 & $-0.51 \%$ & +0.75 & $+3.17 \%$ & 0.50 & $2.60 \%$ \\
\hline
\end{tabular}




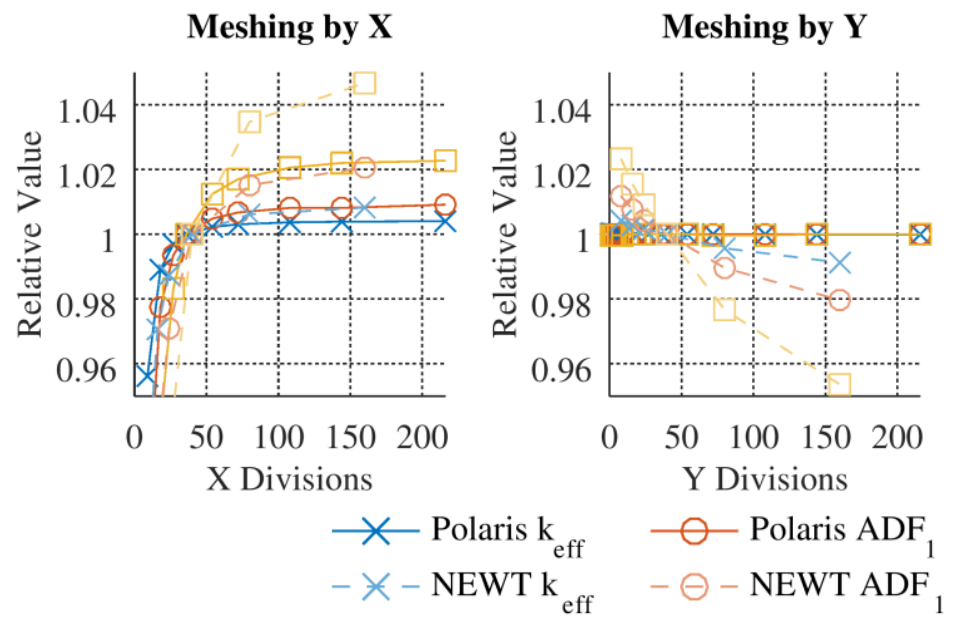

Meshing by X and Y (square mesh)

Figure 2. Convergence of Reflector Model with Mesh Size

\subsection{Correlation study using Polaris and TRITON-NEWT with Sampler}

When comparing the results of Polaris and TRITON for a depletion calculation, $\mathrm{k}_{\infty}$ and fuel temperature coefficient are well-correlated, though Fig. 3 shows two distinct trendlines for the FTC, separated by about $0.08 \mathrm{pcm} / \mathrm{K}$, or about $24 \mathrm{pcm}$ for a $300 \mathrm{~K}$ difference. This was found to be due to two bugs - one in Sampler which produces incorrect self-shielding factors in a few isolated cases (i.e. individual entries in the self-shielding factor table), and a second in Polaris in how it handles such incorrect self-shielding factors for the within-group scattering cross-sections when processing the scattering matrix.

The coolant void reactivity is only moderately correlated. This is expected as the coolant void branch has the most significant effect on the self-shielding calculation, which is where the two codes primarily differ. The equivalent pin cell in TRITON will be affected differently by perturbations compared to the full geometry, particularly if structural materials are perturbed. As well, the effect of perturbing nuclear data on the equivalent lattice pitch calculation (using Dancoff factors) is not captured. It is currently not possible to produce a continuous-energy Monte Carlo reference using Sampler, as support for perturbing the nuclear data for such a calculation is not available as of this writing.
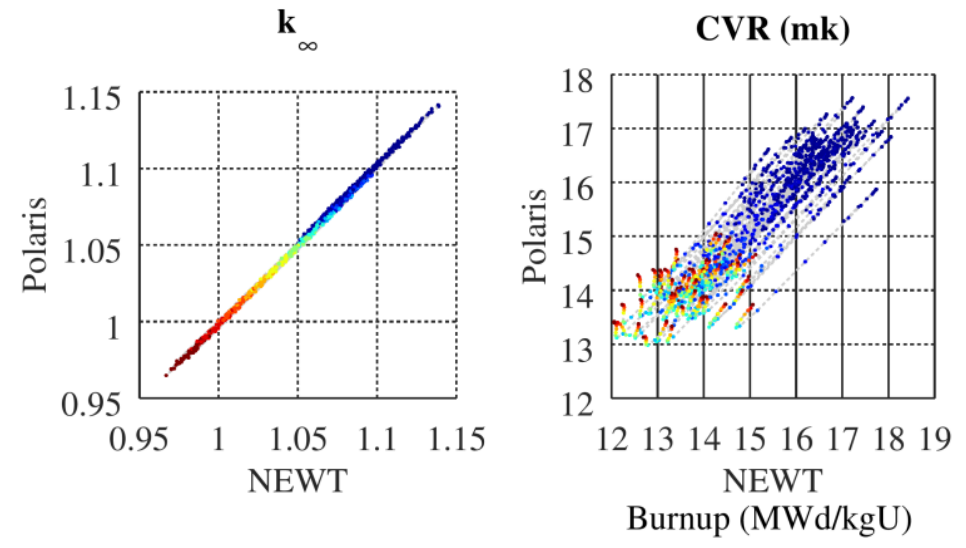

4

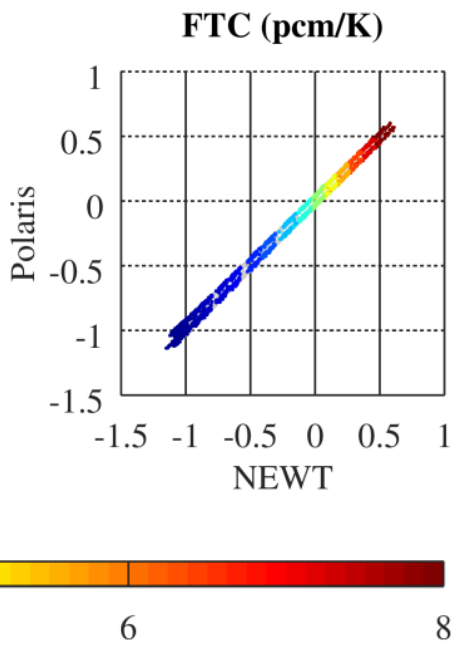

8

Figure 3. Correlation Between TRITON-NEWT and Polaris for a CANDU Depletion Model 
Another useful comparison is on how the uncertainty calculation is affected by model parameters, such as mesh detail. Since computational requirements scale linearly with the number of desired samples, a Sampler calculation on a detailed model can be quite expensive. However, if it is possible to calculate very similar uncertainties using a less detailed model, a lot of time can be saved.

The results in Fig. 4 show that the correlation when considering both depletion and nuclear data uncertainty are nearly perfect when the mesh density is reduced by a factor of 3 to 4 . The uncertainty calculations themselves differ by less than $2 \%$. A bias of roughly $20 \mathrm{pcm}$ is observed for the coolant void reactivity. This indicates that uncertainty calculations can be carried out with a less detailed model compared to the "best estimate" calculation. Polaris has an advantage here, as TRITON is potentially bottlenecked by self-shielding calculations when using CENTRM.

$\mathbf{k}_{\infty}$

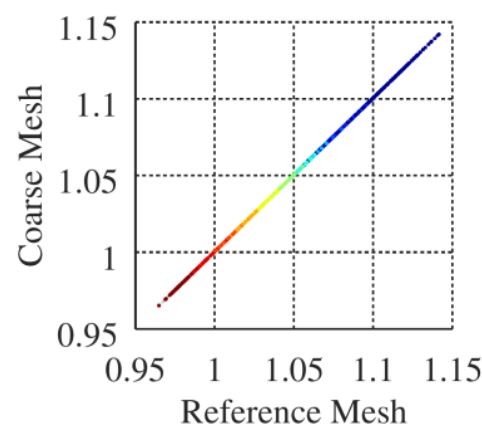

CVR (mk)

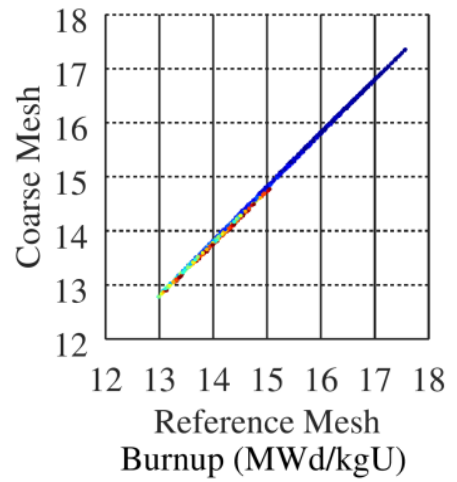

FTC $(\mathbf{p c m} / \mathbf{K})$

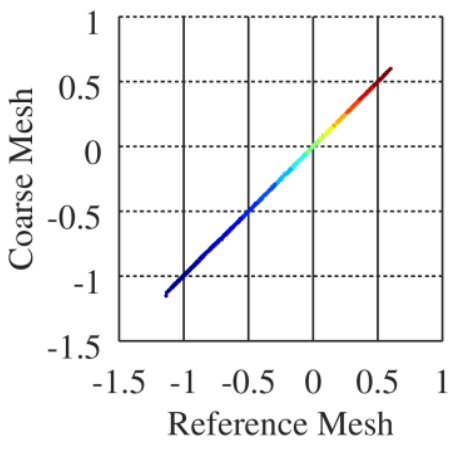

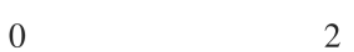

4

6 8

Figure 4. Correlation for Polaris Mesh Size for a CANDU Depletion Model ( $1 \mathrm{mk}=100 \mathrm{pcm})$

\subsection{Investigation of CENTRM mass lumping for self-shielding factors}

Table 2 shows a comparison of the bias, due to self-shielded cross-sections, for two quantities, the first being $\mathrm{k}_{\infty}$, and the second being the ratio of the ${ }^{238} \mathrm{U}$ radiative capture reaction and total fission. A ratio is used to normalize the reaction rate, with fission rate being approximately proportional to fission power. This is separated into the effect of the thermal cross-sections (below $0.625 \mathrm{eV}$ ) and resolved resonance cross-sections (from $0.625 \mathrm{eV}$ up to $20 \mathrm{keV}$ ). The results from cross-sections above $20 \mathrm{keV}$ are insignificant. The overall result is that changing the alump parameter from 0.2 to 0 has virtually no effect when the CENTRM pin cell is moderated by light water, but has a significant effect when the CENTRM pin cell is moderated by heavy water. This is also shown by the effect on depletion trajectories, depicted in Fig. 5. This suggests that the default alump $=0.2$ should not be used for heavy water moderated pin cells, or perhaps for any moderator other than light water, whether for running a TRITON model or for generating heavy water moderated self-shielding factors for Polaris.

There are also significant differences (not shown) between the $\mathrm{H}_{2} \mathrm{O}$ f-factor model used here and the originally distributed one. This provides evidence that the development of models for the self-shielding factor generation shall be done with care, as the choice of reference CENTRM models can noticeably impact the transport results.

The $\mathrm{D}_{2} \mathrm{O}$ pin cell library with alump=0 produces very similar results to the $\mathrm{D}_{2} \mathrm{O}$ homogeneous mixture library for depletion. 
Table 2. Comparison of biases resulting from self-shielded cross-section treatment

\begin{tabular}{|c|c|c|c|c|}
\hline Method/Library & $\mathrm{k}_{\infty}$ Thermal & $\mathrm{k}_{\infty}$ Resonance & ${ }^{238} \mathrm{U}(\mathrm{n}, \gamma)$ Thermal & ${ }^{238} \mathrm{U}(\mathrm{n}, \gamma)$ Resonance \\
\hline CENTRM (alump $=0.2)$ & $+39.9 \mathrm{pcm}$ & $-31.6 \mathrm{pcm}$ & $-0.5137 \%$ & $+0.1208 \%$ \\
\hline CENTRM (alump $=0.0)$ & $+13.7 \mathrm{pcm}$ & $-28.6 \mathrm{pcm}$ & $-0.0180 \%$ & $+0.1155 \%$ \\
\hline $\mathrm{H}_{2} \mathrm{O}$ Homogeneous & $+34.9 \mathrm{pcm}$ & $+3.1 \mathrm{pcm}$ & $-0.3180 \%$ & $+0.0009 \%$ \\
\hline $\mathrm{D}_{2} \mathrm{O}$ Homogeneous & $+24.5 \mathrm{pcm}$ & $+1.1 \mathrm{pcm}$ & $-0.2001 \%$ & $+0.0058 \%$ \\
\hline $\mathrm{D}_{2} \mathrm{O}$ F-Factors (alump $\left.=0.2\right)$ & $+99.6 \mathrm{pcm}$ & $-8.4 \mathrm{pcm}$ & $-0.8421 \%$ & $+0.0281 \%$ \\
\hline $\mathrm{D}_{2} \mathrm{O}$ F-Factors (alump $\left.=0.0\right)$ & $+49.4 \mathrm{pcm}$ & $-9.0 \mathrm{pcm}$ & $-0.3167 \%$ & $+0.0311 \%$ \\
\hline $\mathrm{H}_{2} \mathrm{O}$ F-Factors (alump $\left.=0.2\right)$ & $+132.9 \mathrm{pcm}$ & $-0.7 \mathrm{pcm}$ & $-0.6573 \%$ & $-0.0017 \%$ \\
\hline $\mathrm{H}_{2} \mathrm{O}$ F-Factors (alump $\left.=0.0\right)$ & $+132.9 \mathrm{pcm}$ & $-0.8 \mathrm{pcm}$ & $-0.6573 \%$ & $-0.0016 \%$ \\
\hline
\end{tabular}

\section{U-235}

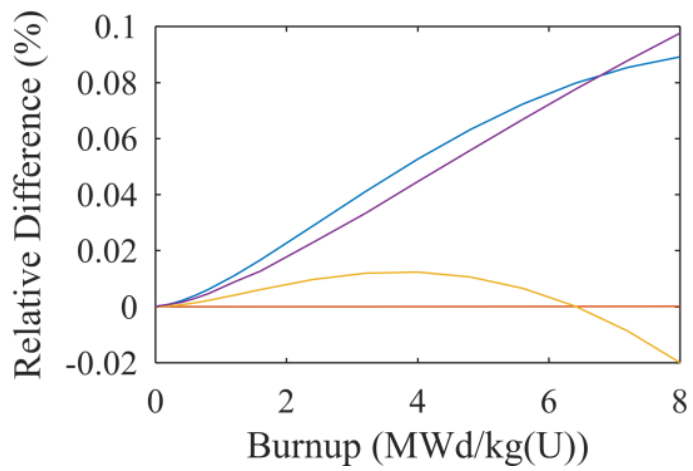

Polaris, D O Pin Lib: Set alump $=0$

Polaris, D 2 O Pin Lib vs. Homog. Lib
Pu-239

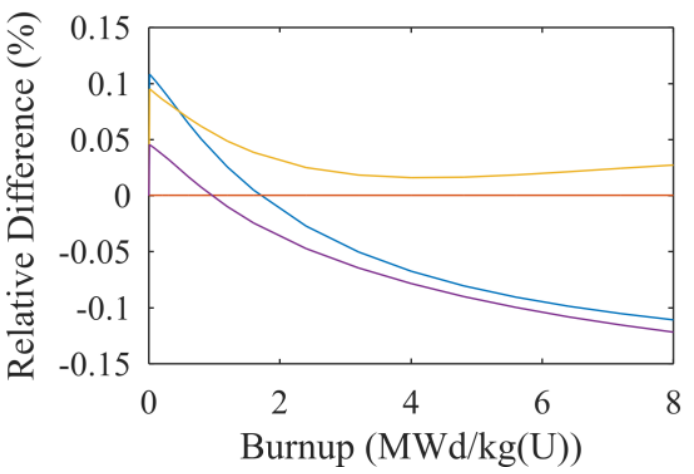

Polaris, $\mathrm{H}_{2} \mathrm{O}$ Pin Lib: Set alump $=0$

NEWT, CENTRM: Set alump $=0$

Figure 5. Effect of Library and Parameter Changes on Depletion Trajectories

\section{CONCLUSIONS}

Overall, Polaris can produce results comparable to those of other SCALE lattice physics sequences, despite its much quicker self-shielding method. The reflector calculation produces similar results between NEWT and Polaris, with much of the difference being attributable to the selection of mesh density. The two packages are well-correlated for $\mathrm{k}_{\infty}$ and FTC, and reasonably correlated for CVR. Finally, this study finds that the default CENTRM mass lumping parameter is ill-suited for heavy water moderated systems, as setting this parameter to zero produces a significant change in results.

This study has identified an issue in the scattering matrix processing in Polaris which resulted in noticeable discontinuous behavior in the FTC results in Fig. 3. The processing of such cases should be corrected for the next SCALE release. However, it is recommended that the underlying issue with Sampler be corrected, to avoid producing bad entries in the self-shielding factor tables in the first place.

While setting alump $=0$ for $\mathrm{D}_{2} \mathrm{O}$ pin cells "corrects" the discrepancy between self-shielding factors generated using pin cells versus homogeneous mixtures, there is still no clearly superior selection for the self-shielding factors. For the SCALE 252-group library, the default library should be adequate for CANDU models. If custom self-shielding factors are generated, merely changing the moderator might not be sufficient, as a careful selection of reference pin cell models, to best match the full 2D model, may have just as significant of an effect on the self-shielding calculation. 


\section{FUTURE WORK}

One key area for future study is a comparison against additional lattice physics codes, particularly nonSCALE codes, including Monte Carlo codes such as Serpent and MCNP or codes developed for CANDU analysis such as DRAGON and WIMS-AECL.

\section{NOMENCLATURE}

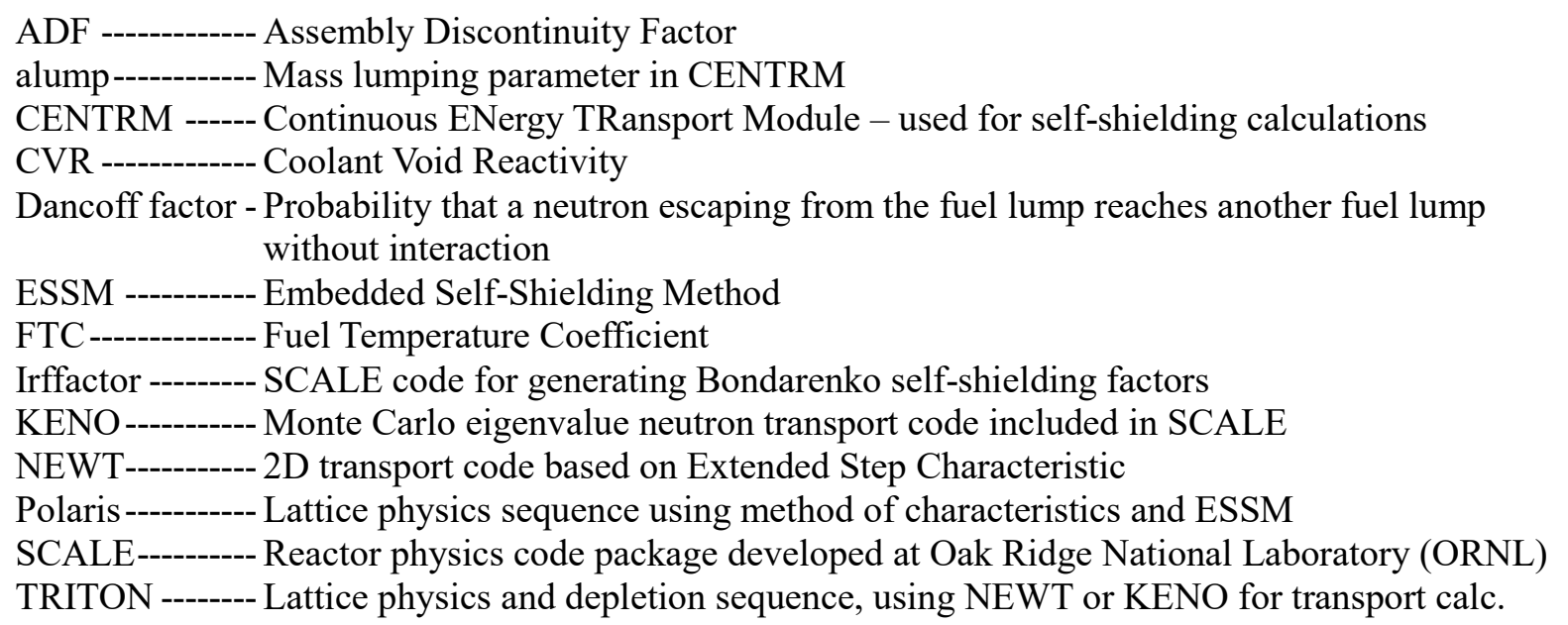

\section{ACKNOWLEDGMENTS}

I would like to acknowledge my supervisor, Dr. Novog, for supporting me through this project. I would also like to acknowledge the SCALE development team, and in particular Will Wieselquist and Matthew Jessee, for their work with the SCALE package and Polaris code, without which this work would not be possible.

\section{REFERENCES}

1. Oak Ridge National Laboratory, "SCALE Code System," US Department of Energy, Oak Ridge (2017).

2. M. A. Jessee, W. A. Wieselquist, T. M. Evans, S. P. Hamilton, J. J. Jarrell, K. S. Kim, J. P. Lefebvre, R. A. Lefebvre, U. Mertyurek, A. B. Thompson and M. L. Williams, "Polaris: a new two-dimensional lattice physics analysis capability for the SCALE code system," Oak Ridge National Lab, Oak Ridge (2014).

3. J. E. Atfield, "28-Element Natural UO2 Fuel Assemblies in ZED-2," Nuclear Energy Agency, Report Number NEA/NSC/DOC(2006)1, Identification Number ZED2-HWR-EXP-001 CRIT (2011).

4. S. Younan and D. Novog, "Extension and Preliminary Validation of the Polaris Lattice Physics Code for CANDU Analysis," Nuclear Engineering \& Design, 2020. doi:10.1016/j.nucengdes.2020.110540

5. A. Hébert, "DRAGON5 and DONJON5, the contribution of École Polytechnique de Montréal to the SALOME platform", invited paper presented at the Third Int. Conf. on Physics and Technology of Reactors and Applications (PHYTRA3), May 12 - 14, 2014, Tetouan, Morocco (2014).

6. A. Hébert and A. Santamarina, "Refinement of the Santamarina-Hfaiedh Energy Mesh Between 22.5 $\mathrm{eV}$ and $11.4 \mathrm{keV}$," paper presented at the Int. Conf. on the Physics of Reactors, September 14 - 19, Interlaken, Switzerland (2008). 